\title{
Research
}

Jennifer A Hirst, Nathan Hill, Chris A O'Callaghan, Daniel Lasserson, Richard J McManus, Emma Ogburn, José M Ordóñez Mena, Brian Shine, Clare J Taylor, Maria DLA Vazquez-Montes, Yaling Yang and FD Richard Hobbs

\section{Prevalence of chronic kidney disease in the community using data from OxRen:}

\author{
a UK population-based cohort study
}

\begin{abstract}
Background

Chronic kidney disease (CKD) is a largely

asymptomatic condition of diminished ren

function, which may not be detected until

advanced stages without screening.
\end{abstract}

\section{Aim}

To establish undiagnosed and overall CKD prevalence using a cross-sectional analysis.

\section{Design and setting}

Longitudinal cohort study in UK primary care.

\section{Method}

Participants aged $>60$ years were invited to attend CKD screening visits to determine whether they had reduced renal function lestimated glomerular filtration rate $\left[\right.$ GGFR] $<60 \mathrm{ml} / \mathrm{min} / 1.73 \mathrm{~m}^{2}$ or albumin:creatinine ratio $\geq 3 \mathrm{mg} / \mathrm{mmol}$. Those with existing CKD, low eGFR, evidence of albuminuria, or two positive screening tests attended a baseline assessment (CKD cohort).

\section{Results}

A total of 3207 participants were recruited and 861 attended the baseline assessment. The CKD cohort consisted of 327 people with existing CKD, 257 people with CKD diagnosed through screening (CKD prevalence of $18.2 \%$, $95 \%$ confidence interval $[\mathrm{Cl}]=16.9$ to 19.6 ), and 277 with borderline/transient decreased renal function. In the CKD cohort, $54.4 \%$ were female, mean standard deviation (SD) age was 74.0 SDD 6.9) years, and mean eGFR was 58.0 (SD 18.4 $\mathrm{m} / \mathrm{min} / 1.73 \mathrm{~m}^{2}$. Of the 584 with confirmed CKD, $44.0 \%$ were diagnosed through screening. Over half of the CKD cohort (51.9\%, 447/861) fell into CKD stages 3-5 at their baseline assessment, giving an overall prevalence of CKD stages 3-5 of $13.9 \%(95 \% \mathrm{Cl}=12.8$ to 15.1$)$. More people had reduced eGFR using the Modification of Diet in Renal Disease (MDRD) equation than with CKD Epidemiology Collaboration (CKD-EPI) equation in the 60-75-year age group and more had reduced eGFR using CKD-EPI in the $\geq 80$-year age group

\section{Conclusion}

This study found that around $44.0 \%$ of people living with CKD are undiagnosed without screening, and prevalence of CKD stages 1-5 was $18.2 \%$ in participants aged $>60$ years. Follow-up will provide data on annual incidence, rate of CKD progression, determinants of rapid progression, and predictors of cardiovascular events.

\section{Keywords}

chronic kidney diseases; glomerular filtration rate; kidney failure; OxRen; prevalence; primary health care.

\section{INTRODUCTION}

Chronic kidney disease (CKD) is a largely asymptomatic condition of diminished renal function. ${ }^{1}$ It has an estimated prevalence in England of $15 \%$ in people aged $\geq 35$ years, and an estimated annual cost to the NHS of 1.4 billion GBP. ${ }^{2}$ CKD prevalence increases with age and it is independently associated with increased cardiovascular disease risk and all-cause mortality. ${ }^{3}$ Having CKD stage $\geq 3$ is the leading risk factor for progression to end-stage renal disease (ESRD) ${ }^{4}$ and a strong predictor of hospitalisation. ${ }^{5}$ Globally, CKD mortality has increased by one-third over the last 10 years, accounting for 1.2 million deaths annually worldwide. ${ }^{6}$

International guidelines, such as those from the National Institute for Health and Care Excellence (NICE) ${ }^{7}$ and Kidney Disease: Improving Global Outcomes (KDIGO), ${ }^{8}$ recommend that people with CKD are identified early to address cardiovascular risk, slow CKD progression, and reduce the numbers reaching ESRD and associated costs. ${ }^{9}$ Many patients in primary care have CKD that is not formally recognised, and it is

JA Hirst, DPhil, senior primary care researcher JM Ordóñez Mena, PhD, medical statistician CJ Taylor, MBE, MA, MPH, PhD, FRCGP

National Institute for Health Research (NIHR) academic clinical lecturer; Y Yang. PhD, senior researcher in health economics, FDR Hobbs, MA, FRCP, FRCPE, FESC, FRCGP, FMedSci, head of department; Nuffield Department of Primary Care Health Science; NIHR Oxford Biomedical Research Centre; CA O'Callaghan, $\mathrm{BM}, \mathrm{BCh}, \mathrm{MA}, \mathrm{DM}, \mathrm{DPhil}, \mathrm{FRCP}$, professor of medicine and consultant nephrologist, Nuffield Department of Medicine; RJ McManus, PhD, FRCGP, professor of primary care research, Nuffield Department of Primary Care Health Science; E Ogburn, $\mathrm{PhD}$, director of operations, Nuffield Department of Primary Care Health Science; MDLA Vazquez-Montes, PhD, senior statistician, Nuffield Department of Primary Care Health Science, University of Oxford, Oxford. N Hill, DPhil, regional access manager, Bristol Myers Squibb Pharmaceuticals Ltd. D Lasserson unclear whether this has any bearing on their prognosis. $^{10}$

CKD is characterised by measures of renal function including increasing urinary albumin:creatinine ratio (ACR) or decreasing glomerular filtration rate (GFR), estimated from serum creatinine (eGFR). In line with recommendations from $\mathrm{NICE}^{7}$ and $\mathrm{KDIGO}{ }^{8}$ CKD is classified into stages based on eGFR and ACR cut-offs. Figure 1, adapted from KDIGO guidelines, ${ }^{8}$ shows the categories and increased risk of adverse outcomes as CKD stage increases. Many reports of CKD prevalence have only used a single measurement, ${ }^{11}$ which may have resulted in biased estimates.

Though CKD is usually asymptomatic until later stages of the disease (stage $\geq 4$ ), earlier stages (1-3) are associated with increased mortality ${ }^{12}$ and cardiovascular risk. ${ }^{3}$ It may therefore be beneficial to establish efficient detection mechanisms for patients with early-stage CKD. Screening enables early detection, but mass screening of all age groups for kidney disease is expensive, of low yield, and not cost-effective. ${ }^{13}$ There is an agerelated decline in renal function and UK data

MD, MRCGP, FRCPE, professor of ambulatory care, University of Birmingham, Birmingham. B Shine, MB ChB, MD, MSc, FRCPath, consultant chemical pathologist, NIHR Oxford Biomedical Research Centre, Oxford University Hospitals NHS Foundation Trust, Oxford.

\section{Address for correspondence}

FDR Hobbs, Nuffield Department of Primary Care Health Sciences, University of Oxford, Radcliffe Observatory Quarter, Woodstock Road, Oxford OX2 6GG, UK.

Email: jennifer.hirstaphc.ox.ac.uk; richard.hobbsda phc.ox.ac.uk.

Submitted: 16 July 2019; Editor's response: 9 August 2019; final acceptance: 30 August 2019. CThe Authors

This is the full-length article (published online $11 \mathrm{Feb} 2020$ ) of an abridged version published in print. Cite this version as: $\mathbf{B r} \mathbf{J}$ Gen Pract 2020; DOI: https://doi.org/10.3399/bjgp20X708245 


\section{How this fits in}

Chronic kidney disease (CKD) is a largely asymptomatic condition with a reported prevalence of around 15\% in England in people aged $\geq 35$ years. Routine screening in older people may detect undiagnosed CKD. Using two measurements of serum creatinine and urinary albumin, this study found the prevalence was $18.2 \%$ for anystage CKD and $13.9 \%$ in stages $3-5$ in an older primary care population laged $\geq 60$ years). Screening using these two measurements identified that around $8 \%$ of older people were living with undiagnosed CKD.

Figure 1. Classification of chronic kidney disease (CKD) using glomerular filtration rate (GFR) and albumin:creatinine ratio (ACR).

Adapted from $K D I G O$ guidelines ${ }^{8}$ with permission from Elsevier. $A 1$ = normal albuminuria. $A 2=$ micro albuminuria. $A 3=$ macro-albuminuria. $G=$ stage of CKD (1-5) by GFR category. show that from age 60 years the prevalence of CKD stages 3-5 increases rapidly. ${ }^{14,15}$ it may, therefore, be reasonable to consider screening systematically in this age group.

The Oxford Renal Longitudinal Cohort Study (OxRen) is a prospective observational longitudinal cohort study of a primary care

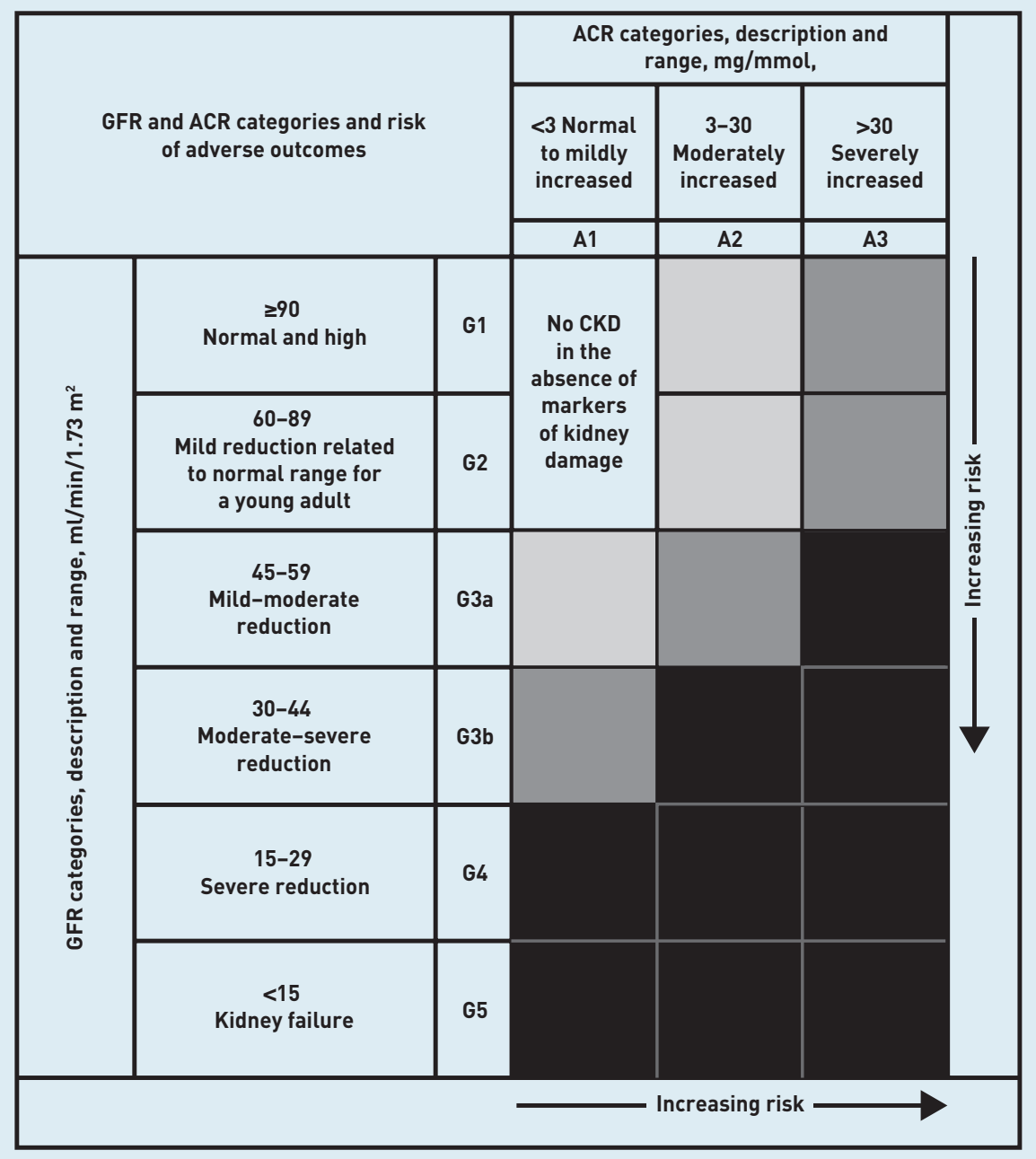

population aged $\geq 60$ years. The study was established by the University of Oxford in 2013 in the central region of the UK to answer important research questions around these uncertainties. The key objectives were to determine the prevalence, annual incidence, and progression of CKD in an older UK population to define the prevalence of selected risk factors and establish the distribution of estimated kidney function in patients detected by a targeted screening programme. ${ }^{16}$ This article reports estimates of the proportion of people with undiagnosed CKD, overall CKD prevalence, and characteristics from the OxRen study cohort.

\section{METHOD}

Participants aged $\geq 60$ years were recruited in 13 primary care clinics across Oxfordshire, UK, over 44 months, from November 2013 to July 2017. Patients were excluded if they were unable to give informed consent, were terminally ill, had a previous solid organ transplant, or if the GP judged them to be unable to give consent. Eligible participants were invited to attend an initial visit where the study was explained and written informed consent was obtained.

Participants entered the OxRen CKD cohort either on the basis of an existing diagnosis of CKD in their GP records, or through screening. Screening visits involved a serum creatinine test to calculate eGFR and a urinary ACR test. Participants with no previous diagnosis of CKD attended a screening visit (A). If the screening result showed decreased renal function leGFR $<65 \mathrm{ml} / \mathrm{min} / 1.73 \mathrm{~m}^{2}$ ) or evidence of kidney damage (ACR $\geq 3 \mathrm{mg} / \mathrm{mmol}$ ), the participant proceeded to a second screening visit (B) after a 3-month interval to have their CKD diagnosis confirmed. If after this visit, the participant had two abnormal results leGFR $<60 \mathrm{ml} / \mathrm{min} / 1.73 \mathrm{~m}^{2}$ or $A C R \geq 3 \mathrm{mg} / \mathrm{mmol}$ ), they were classified as having CKD and invited to attend the baseline assessment visit at their GP practice.

If on the second occasion the eGFR and ACR did not fall within the criteria for CKD diagnosis, participants were still invited to the baseline assessment visit but classified as having borderline/transient decreased renal function providing their first result suggested CKD stage 1-5. Participants who had a borderline result in their first test and abnormal second test result were also classified as having borderline/transient decreased renal function and invited to attend a baseline assessment. All participants invited to the baseline assessment are hereafter called the CKD cohort (Figure 2). 


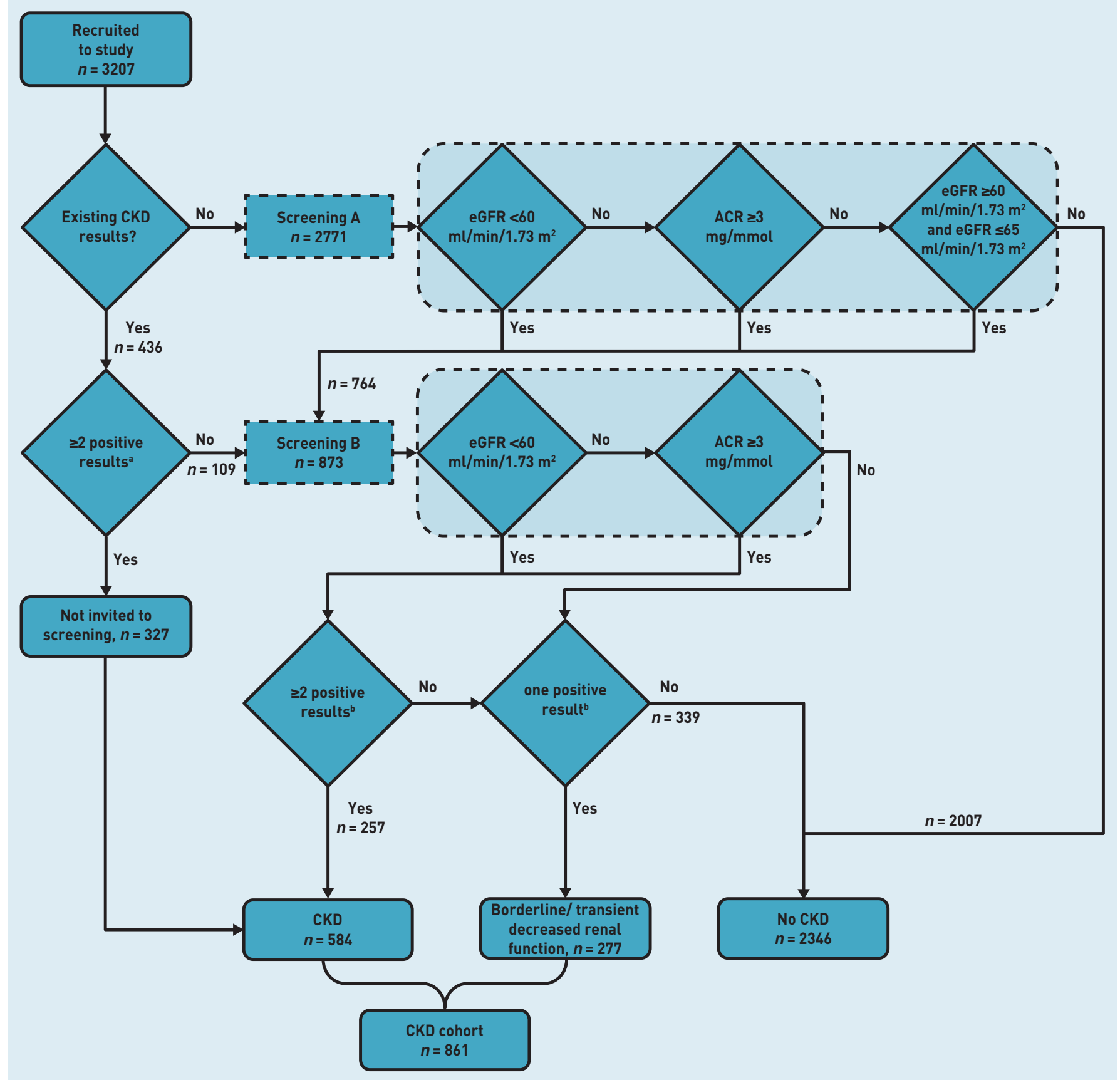

Figure 2. Participant flow chart.

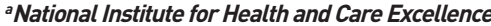
criteria for diagnosis of chronic kidney disease (CKD): $\geq 2$ results at least 3 months apart. ${ }^{b}$ Estimated glomerular filtration rate (eGFR) $<60 \mathrm{ml} / \mathrm{min} / 1.73 \mathrm{~m}^{2}$, or eGFR $>60 \mathrm{ml} / \mathrm{min} / 1.73 \mathrm{~m}^{2}$, and albumin:creatinine ratio $(A C R)>3 \mathrm{mg} / \mathrm{mmol}$; at least one result must be from Screening $A$ or Screening $B$.
At the baseline assessment visit, data were collected on demographics, lifestyle, and medical history. Anthropometric and clinical measurements (blood pressure and pulse wave velocityl were made. Questionnaires were completed on diet, physical activity, cognitive function, and quality of life. Blood and urine samples were sent for immediate laboratory analysis, and additional blood and urine samples were collected and stored at $-80^{\circ} \mathrm{C}$ for future genetic and protein testing.

All samples were processed across two laboratories using identical instrumentation and assay standardisation. Serum creatinine was measured using an isotope dilution mass spectrometry traceable method ${ }^{17}$ and eGFR was reported using the Modification of Diet in Renal Disease (MDRD) equation. ${ }^{18}$ The most recent test result was used for 12 participants where no results were available at the time of the test. The effect of these approximations on results was tested by re-analysing data excluding these participants from the analysis.

\section{Data management and reporting}

Participants whose laboratory eGFR was reported as $>90 \mathrm{ml} / \mathrm{min} / 1.73 \mathrm{~m}^{2}(n=2)$ were 
designated to have eGFR of $91 \mathrm{~mL} / \mathrm{min} / 1.73 \mathrm{~m}^{2}$. Urinary albumin levels were approximated to $4.9 \mathrm{mg} / \mathrm{L}$ for 29 participants whose laboratory urinary albumin levels were reported as $<5 \mathrm{mg} / \mathrm{L}$. These were excluded in a sensitivity analysis in two cases where this may have changed stage of CKD. CKD stage was reported for all participants. Results were tabulated for the full CKD cohort and stratified both by stage of CKD and how the participant entered the cohort (previous diagnosis, new diagnosis, or borderline/transient decreased renal function) to report prevalence and 95\% confidence intervals (Cls). ${ }^{19,20}$ The eGFR results and stage of CKD were also reported using the CKD Epidemiology Collaboration (CKD-EPI) equation, which is the global standard for estimating GFR for comparison purposes, and prevalence of reduced eGFR $\left(<60 \mathrm{ml} / \mathrm{min} / 1.73 \mathrm{~m}^{2}\right)$ was compared with MDRD across participant age.

All data management and analyses were carried out using Stata (version 14SE). Data are presented in tables and summarised as number and per cent, or mean and standard deviation (SD). For continuous variables that were non-normally distributed, the median and interquartile range (IQR) are reported.

\section{Data sharing}

Requests to access OxRen data should be addressed to the corresponding authors.

\section{Patient and public involvement}

A patient representative sits on the Study Steering Committee that meets on a 6-monthly basis to assess the progress of participant recruitment and follow-up, to review and contribute to study materials, and to oversee the ethical and safe conduct of the research.

\section{RESULTS}

In total, 8268 people were invited to participate in OxRen and 3207 were recruited (38.8\% recruitment rate). Among recruited participants, 327 had CKD previously documented from GP records and 2880 attended screening visits. At screening, 534 (18.5\%, 534/2880) had one or more positive tests for CKD $(257$ newly diagnosed, 277 borderline/transient decreased renal function). The resulting total of 861 participants (26.8\% of those recruited) entered the CKD cohort and attended the baseline assessment. Overall, newly diagnosed CKD was 8.0\% (257/3207) (95\% $\mathrm{Cl}=7.1$ to 9.0$)$ and pre-existing $\mathrm{CKD}$ was $10.2 \%(327 / 3207)(95 \% \mathrm{Cl}=9.2$ to 11.3$)$ giving CKD prevalence of $18.2 \%$ (584/3207) (95\% $\mathrm{Cl}=16.9$ to 19.6 ). Of the 584 with confirmed
CKD, 44.0\% were diagnosed through screening (Figure 2).

Characteristics of the 861 participants who attended a baseline study visit are shown in Table 1. Results are presented for all participants and stratified by whether they had existing CKD, newly diagnosed, or borderline/ transient decreased renal function. Briefly, $54.4 \%$ were female, mean (SD) age was 74.0 (SD 6.9) years, mean BMI was 28.1 (SD 7.3) kg/m², and 846 (98.3\%) were white. Prevalence of hypertension was $59.3 \%$ and diabetes was $15.4 \%$. These, and prevalence of other comorbidities, are presented for the full cohort and for different subgroups in Tables 1 and 2, and Supplementary Table S1.

Stratification by stage of CKD using eGFR and ACR shows that 258 participants (30.0\%) met the KDIGO definition of normal kidney function (eGFR $\geq 60 \mathrm{ml} / \mathrm{min} / 1.73 \mathrm{~m}^{2}$ and ACR $<3 \mathrm{mg} / \mathrm{mmol}), 83$ participants (4.0\%) had CKD stage 1, 122 (14.2\%) had CKD stage 2, 246 (28.6\%) had CKD stage 3a, 133 (15.5\%) had CKD stage 3b, 67 $(7.8 \%)$ had CKD stage 4 , and one $(0.12 \%)$ had stage 5 CKD. Over half of the CKD cohort fell into CKD stages 3-5, which gave a prevalence of CKD stages $3-5$ of $13.9 \%$ (447/3207) from the full population screened (see Table 2 and Supplementary Table S1). Results were similar in sensitivity analyses where approximations were made (see Supplementary Table S2 for details).

CKD stage of participants stratified by entry to the CKD cohort is shown in Figure 3 and Supplementary Table S3. Sixty-five participants with previously diagnosed CKD (20\%, N=327), 58 with newly diagnosed CKD (23\%, N=257), and 135 with borderline/transient decreased renal function $(49 \%, N=277)$ were classified as having normal kidney function (by KDIGO definition $)^{8}$ at their baseline visit. Stage of CKD and eGFR calculated using the CKDEPI equation is shown in Supplementary Table S4 and S5, and Supplementary Figure S1. Overall, there were more people with CKD stages 2 and 4 and fewer people with CKD stages $1,3 a$, and $3 b$ compared with CKD stage calculated using the MDRD equation. More people had reduced eGFR $\left(<60 \mathrm{ml} / \mathrm{min} / 1.73 \mathrm{~m}^{2}\right)$ using MDRD than with CKD-EPI in the 60-75-year age group, and more had reduced eGFR using CKD$\mathrm{EPI}$ in the $\geq 80$-year age group (Figure 4 ).

\section{DISCUSSION}

\section{Summary}

This large-scale screening study from UK primary care offers a prevalence estimate of $18.2 \%(95 \% \mathrm{Cl}=16.9$ to 19.6$)$ for $\mathrm{CKD}$ stages $1-5$ in people aged $\geq 60$ years and has found 
Table 1. Patient demographics stratified by screening data

\begin{tabular}{|c|c|c|c|c|}
\hline Characteristics & $\begin{array}{l}\text { Full cohort } \\
\qquad(N=861)\end{array}$ & $\begin{array}{l}\text { Existing CKD } \\
\qquad(N=327)\end{array}$ & $\begin{array}{l}\text { Newly diagnosed CKD } \\
\qquad(N=257)\end{array}$ & $\begin{array}{l}\text { Borderline/transient decreased } \\
\text { renal function }(N=277)\end{array}$ \\
\hline CKD prevalence, $\%(95 \% \mathrm{CI})$ & $18.2(16.9$ to 19.6$)$ & 10.2 (9.2 to 11.3$)$ & 8.0 (7.1 to 9.0 ) & - \\
\hline Mean age, years (SD) & $74.0(6.9)$ & $75.4(7.0)$ & $73.4(6.5)$ & $72.7(6.7)$ \\
\hline Female, $n(\%)$ & $468(54.4)$ & $183(56.0)$ & $124(48.2)$ & $161(58.1)$ \\
\hline Mean weight, kg (SD) & $78.0(17.1)$ & $77.3(16.1)$ & $80.2(18.0)$ & $76.8(17.4)$ \\
\hline Mean height, cm (SD) & $167.0(10.5)$ & $167.0(10.2)$ & $167.8(10.0)$ & $166.1(11.2)$ \\
\hline Mean waist circumference, cm (SD) & $97.0(14.6)$ & $97.0(13.4)$ & $99.0(15.2)$ & $95.0(15.1)$ \\
\hline Mean BMI, kg/m² (SD) & $28.1(7.3)$ & $27.6(5.1)$ & $28.5(6.4)$ & $28.2(9.9)$ \\
\hline \multicolumn{5}{|l|}{ Ethnicity, $n(\%)$} \\
\hline White & $846(98.3)^{\mathrm{a}}$ & 323 (98.8) & 253 (98.4) & $271(97.8)$ \\
\hline Other & $14(1.6)$ & $4(1.2)$ & $4(1.6)$ & $6(2.2)$ \\
\hline \multicolumn{5}{|l|}{ Educational status, $n[\%]$} \\
\hline School up to 16 years & $483(56.1)$ & $183(56.0)$ & $150(58.4)$ & $150(54.2)$ \\
\hline Sixth form school or college & $174(20.2)$ & $57(17.4)$ & $55(21.4)$ & $62(22.4)$ \\
\hline \multicolumn{5}{|l|}{ Smoking status, $n(\%)$} \\
\hline Never & $466(54.1)$ & $171(52.3)$ & 137 (53.3) & $158(57.0)$ \\
\hline Former & $357(41.5)$ & $142(43.4)$ & $111(43.2)$ & 104 (37.5) \\
\hline Current & $38(4.4)$ & $14(4.3)$ & 9 (3.5) & $15(5.4)$ \\
\hline \multicolumn{5}{|l|}{ Alcohol use, units/week } \\
\hline None, $n(\%)$ & $252(29.3)$ & $85(26.0)$ & 88 (34.2) & 79 (28.5) \\
\hline One or more, $n(\%)$ & 609 (70.7) & $242(74.0)$ & $169(65.8)$ & 198 (71.5) \\
\hline Median (IQR) & 2 (0 to 8) & $2(0$ to 7$)$ & $2(0$ to 9$)$ & 3 (0 to 10$)$ \\
\hline Mean (SD) & $5.9(8.7)$ & $5.3(8.0)$ & $6.1(9.1)$ & $6.4(9.0)$ \\
\hline \multicolumn{5}{|l|}{ Major comorbidities, $n[\%]$} \\
\hline Hypertension & 511 (59.3) & $213(65.1)$ & 160 (62.3) & 138 (49.8) \\
\hline Diabetes & 133 (15.4) & $71(21.7)$ & $38(14.8)$ & 24 (8.7) \\
\hline Ischaemic heart disease & $145(16.8)$ & $62(19.0)$ & 50 (19.5) & $33(11.9)$ \\
\hline Heart failure & $39(4.5)$ & $19(5.8)$ & $13(5.1)$ & 7 (2.5) \\
\hline \multicolumn{5}{|l|}{ Systolic blood pressure, $\mathrm{mmHg}$} \\
\hline Median (IQR) & 132 (120 to 144$)$ & $130(119$ to 142$)$ & 135 (123 to 145$)$ & 131 (121 to 145$)$ \\
\hline Mean (SD) & $133(18)$ & $131(17)$ & $135(19)$ & $132(18)$ \\
\hline \multicolumn{5}{|l|}{ Diastolic blood pressure, $\mathrm{mmHg}$} \\
\hline Median (IQR) & $78(71$ to 84$)$ & 77 (70 to 82 ) & 79 (72 to 85) & 78 (71 to 84$)$ \\
\hline Mean (SD) & $78(10)$ & $77(9.9)$ & $79(11)$ & $78(10)$ \\
\hline \multicolumn{5}{|l|}{ Blood and urine test results } \\
\hline \multicolumn{5}{|l|}{ eGFR, $\mathrm{ml} / \mathrm{min} / 1.73 \mathrm{~m}^{2}$} \\
\hline$>60, n(\%)$ & $396(46.0)$ & 84 (25.7) & 135 (52.5) & 177 (63.9) \\
\hline$\leq 60, n(\%)$ & $465(54.0)$ & 243 (74.3) & $122(47.5)$ & $100(36.1)$ \\
\hline Median (IQR) & 59 (46 to 69 ) & 50 (35 to 61$)$ & 61 (48 to 76 ) & $63(57$ to 74$)$ \\
\hline Mean (SD) & $58.0(18.4)$ & $48.9(17.1)$ & $61.4(18.7)$ & $65.7(14.6)$ \\
\hline ACR, $\mathrm{mg} / \mathrm{mmol}, N[\%]$ & 774 (89.9) & $288(88.1)$ & $239(93.0)$ & 247 (89.2) \\
\hline$<3.0, n(\%)$ & 489 (63.2) & $194(67.4)$ & $116(48.5)$ & $214(86.6)$ \\
\hline$\geq 3.0, n(\%)$ & 285 (36.8) & $94(32.6)$ & 123 (51.5) & $148(59.9)$ \\
\hline Median (IQR) & 2.0 (0.9 to 4.8$)$ & 1.6 (0.6 to 4.2$)$ & 3.1 (1.3 to 7.5$)$ & 1.6 (0.9 to 3.4$)$ \\
\hline Mean (SD) & $5.2(11.4)$ & $5.1(12.3)$ & $7.8(14.6)$ & $2.8(3.6)$ \\
\hline \multicolumn{5}{|l|}{ Questionnaires, $n$ completed (\%) } \\
\hline Quality of life & $859(99.8)$ & $327(100.0)$ & $256(99.6)$ & $276(99.6)$ \\
\hline Diet & $310(36.0)$ & $148(45.3)$ & 79 (30.7) & $83(30.0)$ \\
\hline Physical activity & 736 (85.5) & 318 (97.2) & 205 (79.8) & $213(76.9)$ \\
\hline Cognitive assessment & $625(72.6)$ & $249(76.1)$ & $182(70.8)$ & 194 (70.0) \\
\hline
\end{tabular}

ata missing for one patient. $A C R=$ albumin:creatinine ratio. $B M I=$ body mass index. $C l=$ confidence interval. $C K D=$ chronic kidney disease. eGFR = estimated glomerular filtration rate. $I Q R=$ interquartile range. $S D=$ standard deviation. 
Table 2. Patient demographics stratified by CKD stage, $N=860^{a}$

\begin{tabular}{|c|c|c|c|c|c|c|}
\hline Characteristics & $\begin{array}{c}\text { Normal kidney } \\
\text { function ( } N=258)\end{array}$ & $\begin{array}{l}\text { CKD stage } 1 \\
\qquad(N=34)\end{array}$ & $\begin{array}{c}\text { CKD stage } 2 \\
(N=122)\end{array}$ & $\begin{array}{l}\text { CKD stage } 3 a \\
\qquad(N=246)\end{array}$ & $\begin{array}{l}\text { CKD stage } 3 b \\
\qquad(N=133)\end{array}$ & $\begin{array}{c}\text { CKD stage } 4 \\
(N=67)\end{array}$ \\
\hline Mean age, years (SD) & $72.6(6.7)$ & $70.6(4.8)$ & $73.6(7.3)$ & $74.5(6.3)$ & $75.7(7.2)$ & $76.3(7.2)$ \\
\hline Female, $n(\%)$ & 158 (61.2) & 13 (38.2) & $54(44.3)$ & 132 (53.7) & $76(57.1)$ & 34 (50.7) \\
\hline Weight, kg (SD) & $76.6(16.9)$ & $79.9(23.4)$ & 76.6 (17.2) & $78.6(17.2)$ & 79.1 (16.5) & $80.8(15.1)$ \\
\hline Height, cm (SD) & $167.0(10.7)$ & $169.1(9.3)$ & $166.8(10.4)$ & $166.8(10.5)$ & $167.0(9.7)$ & $167.2(12.1)$ \\
\hline Waist circumference, cm (SD) & 95.5 (15.3) & 97.3 (18.5) & $95.1(15.4)$ & $97.5(13.7)$ & $98.6(13.3)$ & $100.8(12.7)$ \\
\hline Hip circumference, cm (SD) & $105.8(11.1)$ & $102.4(10.5)$ & $104.1(9.7)$ & $107.0(9.9)$ & $108.4(11.1)$ & $109.2(11.3)$ \\
\hline $\mathrm{BMI}, \mathrm{kg} / \mathrm{m}^{2}$ (SD) & $27.4(5.8)$ & $27.6(6.6)$ & $27.7(7.7)$ & $28.4(9.1)$ & $28.3(5.4)$ & $29.3(8.3)$ \\
\hline \multicolumn{7}{|l|}{ Ethnicity, $n(\%)$} \\
\hline White & $254(98.4)$ & $34(100.0)$ & $120(98.4)$ & $239(97.2)^{b}$ & 132 (99.2) & 66 (98.5) \\
\hline Other & $4(1.6)$ & - & $2(1.6)$ & 6 (2.4) & $1(0.8)$ & $1(1.5)$ \\
\hline
\end{tabular}

${ }^{a}$ One participant with $C K D$ stage 5 not included. ${ }^{b}$ Data missing for one patient. BMI = body mass index. $C K D=$ chronic kidney disease. $S D=$ standard deviation.

Figure 3. Chronic kidney disease (CKD) stage of participants stratified by entry to the cohort calculated using the Modification of Diet in Renal Disease (MDRD) equation. that $8.0 \%$ of this population had CKD that was previously undiagnosed. More than half $(51.3 \%)$ of those with only a single positive CKD screening test (borderline/ transient decreased renal function group), thus not meeting the diagnostic criteria for CKD, were classified into CKD stages 1-4 at the baseline assessment. Providing the baseline samples were taken a minimum of 12 weeks from their previous positive test, these participants now technically meet the diagnostic criteria for CKD. ${ }^{8}$

\section{Strengths and limitations}

This study is methodologically strong, using both MDRD and CKD-EPI equations to calculate eGFR and including urinary ACR to estimate CKD prevalence unlike many

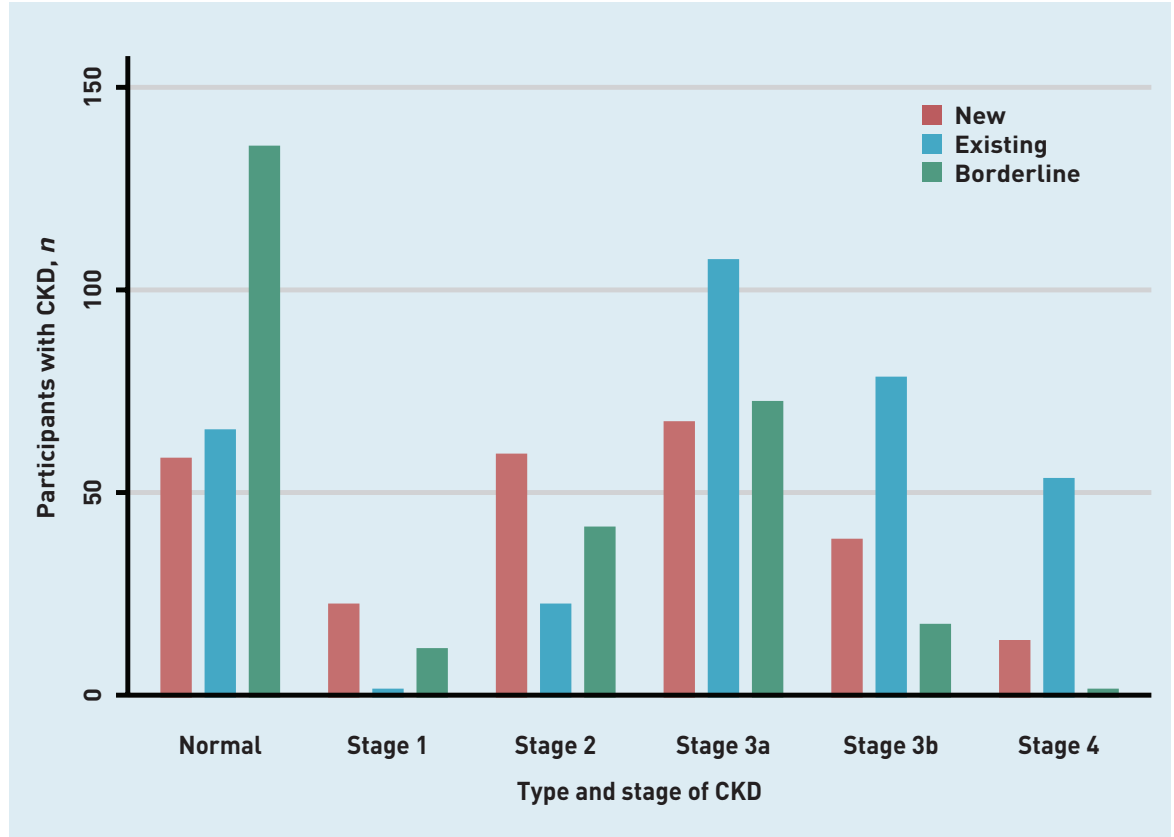

previous registry studies, which have had less urinary ACR data available. This study reports $C K D$ prevalence by stage of $C K D$ and found that more participants were classified into CKD stage 3a than any other CKD stage. Furthermore, over one-fifth (21.1\%) of those with existing CKD were classed as having normal renal function from analysis of blood and urine samples taken at their baseline visit. This highlights how the variability in both eGFR and ACR can result in some participants, who had previously met the diagnostic criteria for CKD, falling into the normal kidney function range on repeat testing. This suggests that there is potential for disease misclassification, which could be an artefact of the interval between screening visits. ${ }^{21}$ As more laboratories move away from MDRD to use other equations for estimating GFR, $7,22,23$ the CKD stage for some individuals may change, and others may no longer meet criteria for diagnosis. ${ }^{22}$ Findings from this study corroborate those from other studies, in that moving to CKD-EPI will result in a greater proportion of people aged $>75$ years having decreased eGFR compared with MDRD and fewer people aged $<75$ years with reduced eGFR. ${ }^{14,24}$ This analysis has also quantified how moving to the CKD-EPI equation may lead to classification into a different CKD stage for some participants.

There are some limitations to this study. It is possible that people who were aware that they had CKD were more likely to agree to participate in the study, which may have led to an overestimation in the prevalence of CKD in this cohort. Over $98 \%$ of the OxRen cohort were white, which is a higher proportion than the 95\% white ethnicity reported in the general UK population aged $\geq 60$ years. ${ }^{25}$ This is also higher than 


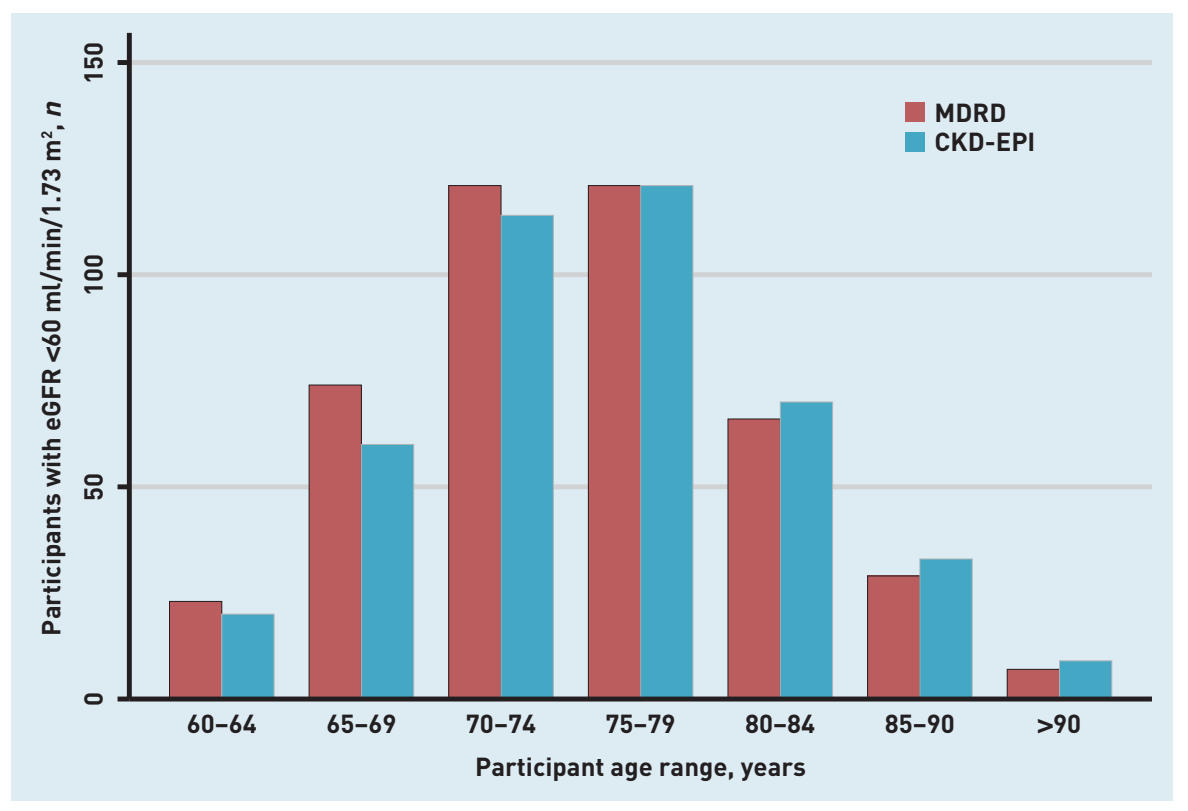

Figure 4. Participant eGFR using the Modification of Diet in Renal Disease (MDRD) equation and the Chronic Kidney Disease Epidemiology Collaboration (CKD-EPI) equation stratified by age. $C K D=$ chronic kidney disease. eGFR = estimated glomerular filtration rate.

\section{Funding}

The research was funded by the National Institute for Health Research (NIHR) Oxford Biomedical Research Centre (OUHT BRC) (grant reference: BRC-1215-20008). Jennifer Hirst and José M Ordóñez Mena are funded by the NIHR Biomedical Research Centre, Oxford. Daniel Lasserson receives funding from the NIHR Community Healthcare MedTECH and In Vitro Diagnostic Cooperative. Clare Taylor is funded by an NIHR Academic Clinical Lectureship. FD Richard Hobbs acknowledges part-support from the NIHR School for Primary Care Research, the NIHR Collaboration for Leadership in Applied Health Research and Care (CLAHRC) Oxford, and the OUHT BRC. Richard McManus acknowledges part-support from the NIHR CLAHRC Oxford. The views expressed are those of the authors and not necessarily those of the NHS, the $\mathrm{NIHR}$, or the Department of Health and Social Care. This work uses data provided by patients and collected by the NHS as part of their care and support, and would not have been possible without access to this data. NIHR recognises and values the role of patient data, securely accessed and stored, both in underpinning and leading to improvements in research and care. The content of this article is solely the responsibility of the authors and does not necessarily represent the official views of the study sponsors. the rest of Oxfordshire where $92 \%$ of the population is white. ${ }^{26}$ The low percentage of ethnic minority participants in the cohort may have affected the CKD prevalence. Some reports suggest that South Asians have a lower prevalence of CKD when compared with white individuals after adjustment for age and sex. ${ }^{27}$ However, Asian and African and Caribbean populations with diabetes have been reported to show the most rapid decline in renal function ${ }^{28}$ and African and Caribbean populations have a high incidence of ESRD. ${ }^{28,29}$ There were relatively low numbers of people with CKD stage 1 . 4 , and 5 in the present cohort, but these low numbers are also seen in the general population. ${ }^{2,30}$

Despite efforts to recruit GP practices in Oxfordshire with different indexes of deprivation profiles to ensure comparability with the general UK population, recruitment took place over a single UK county, which may not be fully representative of the of people with university education or above is $18 \%$ in the UK population aged $>65$ years $^{31}$ compared with $24 \%$ in the OxRen population. However, younger age groups in the general population have a greater proportion achieving the highest level of educational attainment and fewer people leaving school with no qualifications compared with older age groups. ${ }^{31}$ As these populations age, findings from the cohort may be generalisable to these age groups after accounting for lifestyle factors. Both lower education and socioeconomic status are associated with higher prevalence of CKD. ${ }^{32}$ general UK population. The proportion

\section{Comparison with existing literature}

UK prevalence of CKD stages 1-5 in people aged $65-74$ years has been reported to be $19 \%,{ }^{2}$ which is similar to prevalence found in the OxRen cohort. For those $>75$ years of age, prevalence reaches $46 \%{ }^{2}$ A systematic review and meta-analysis reporting global prevalence of CKD stratified by age found a prevalence of CKD stages 1-5 of nearly $28 \%$ in populations with a mean age of $>60$ years and around $34 \%$ in those with a mean age of 70 years. ${ }^{30}$ These are higher than the prevalence of $18 \%$ in the present study, which may reflect differences in the diagnostic criteria used, or prevalence in different ethnic groups and socio-economic status. ${ }^{32}$ Other English data suggest that prevalence of CKD stages 3-5 is 12.0-14.5\% in those aged $65-74$ years. ${ }^{2,33}$ This is similar to the $13.9 \%(95 \% \mathrm{Cl}=12.8$ to 15.1$)$ found in the OxRen cohort despite these surveys using a single eGFR test to classify CKD, which has been shown to overestimate CKD. ${ }^{10,22}$ Internationally, most prevalence estimates in similar age groups are only based on a single test result. 11,34,35 In Europe, prevalence differs between countries and ranges from $24 \%$ in Norway to $41 \%$ in Germany. ${ }^{11}$ In the US, prevalence of stages $3-4$ is $23 \%$, ${ }^{34}$ and stages $1-5$ of $34 \%{ }^{35}$

A recently published analysis of participants in the OxRen cohort 22 found that using a single screening test to diagnose CKD would result in around 25\% over diagnosis because of variability in ACR and eGFR. The results from laboratory analyses at participants' baseline visit show that some participants' CKD stage can revert to normal kidney function despite a recent diagnosis. This was observed in $21 \%$ of OxRen participants who had received a CKD diagnosis but tested as normal kidney function, based on samples taken during their baseline visit. A combination of analytical and biological variability may have contributed to this. ${ }^{36}$ These numbers are consistent with a previous report that found CKD remission rates of $20-30 \%$ in follow-up visits in primary care populations with CKD.37

\section{Implications for research and practice}

This study has found that $8.0 \%$ of participants aged $\geq 60$ years had undiagnosed CKD, equating to $44.0 \%$ of people living with CKD in this population, and would have remained undiagnosed without screening. This suggests that the early stages of CKD (stages 1-3b) may be under-reported in the literature as many people are unaware that they have the condition. ${ }^{10,38}$ In the UK, some of these people will be picked up in 


\section{Ethical approval}

The study protocol was approved by South Central Oxfordshire Research Ethics Committee B (reference number: 13/ SC/0020) and registered with the UK Clinical Research Network (registration number: 36916).

\section{Provenance}

Freely submitted; externally peer reviewed.

\section{Competing interests}

Nathan Hill became an employee of BristolMyers Squibb during the present study. Clare Taylor reports personal fees from Novartis, personal fees from Vifor, and nonfinancial support from Roche, outside of the submitted work. The remaining authors have declared no competing interests.

\section{Acknowledgements}

The authors would like to thank the following for their contributions to the study: Samuel Fatoba for developing the study protocol and setting up the cohort; the OxRen Steering Committee members: Maarten Taal, professor of medicine, Royal Derby Hospital and University of Nottingham (chair); Martin Wood, patient representative; Chris Pugh, consultant nephrologist; Kathryn Griffith, GP; the Primary Care Clinical Trials Unit: Vanshika Sharma; Hannah Swayze; Rebecca Lowe; Sue Smith; Heather Rutter; the data team and nurses in the Primary Care Clinical Trials Unit: Sadie Kelly; Luis Castello; Pippa Whitbread; Karen Madronal; Irene Noel; Bernadette Mundy; Belinda l'Anson; Samantha Squires; and all Principal Investigators from participating GP surgeries.

\section{Open access}

This article is Open Access: CC BY-NC 4.0 licence (http://creativecommons.org/ licences/by-nc/4.0/).

\section{Discuss this article}

Contribute and read comments about this article: bjgp.org/letters

the NHS Health Check Programme Inww. healthcheck.nhs.uk), which is a 5-yearly free check-up available for adults in the UK aged $40-74$ years. However, only $43 \%$ of those invited for an NHS Health Check are reported to attend, ${ }^{39}$ and it is likely that many non-attenders will be those most at risk. ${ }^{40}$

OxRen provides an estimate of undiagnosed CKD in the UK population aged $\geq 60$ years, including screen-detected rates, and will provide future estimates of incidence. Follow-up will provide evidence on the progression of CKD, and, if progression occurs, identify groups that are most likely to progress, which will make it possible to explore associations with comorbidities and treatments. 


\section{REFERENCES}

1. James MT, Hemmelgarn BR, Tonelli M. Early recognition and prevention of chronic kidney disease. Lancet 2010; 375(9722): 1296-1309.

2. NHS Digital. Health Survey for England, 2016. 2017. https://digital.nhs.uk/ data-and-information/publications/statistica//health-survey-for-england/healthsurvey-for-england-2016 (accessed 14 Jan 2020).

3. Gansevoort RT, Correa-Rotter R, Hemmelgarn BR, et al. Chronic kidney disease and cardiovascular risk: epidemiology, mechanisms, and prevention. Lancet 2013; 382(9889): 339-352.

4. Keith DS, Nichols GA, Gullion CM, et al. Longitudinal follow-up and outcomes among a population with chronic kidney disease in a large managed care organization. Arch Intern Med 2004; 164(6): 659-663.

5. Go AS, Chertow GM, Fan D, et al. Chronic kidney disease and the risks of death, cardiovascular events, and hospitalization. N Engl J Med 2004; 351(13): 1296-1305.

6. GBD 2015 Mortality and Causes of Death Collaborators. Global, regional, and national life expectancy, all-cause mortality, and cause-specific mortality for 249 causes of death, 1980-2015: a systematic analysis for the Global Burden of Disease Study 2015. Lancet 2016; 388(10053): 1459-1544

7. National Institute for Health and Care Excellence. Chronic kidney disease in adults: assessment and management. CG182. London: NICE, 2014. https:// www.nice.org.uk/guidance/cg182 (accessed 14 Jan 2020).

8. Kidney disease: improving global outcomes (KDIGO) CKD work group. KDGIGO 2012 clinical practice guideline for the evaluation and management of chronic kidney disease. Kidney Int Suppl 2013; 3(1): 1-150.

9. Joyce AT, lacoviello JM, Nag S, et al. End-stage renal disease-associated managed care costs among patients with and without diabetes. Diabetes Care 2004; 27(12): 2829-2835

10. Jain P, Calvert M, Cockwell P, McManus RJ. The need for improved identification and accurate classification of stages $3-5$ chronic kidney disease in primary care: retrospective cohort study. PLoS One 2014; 9(8): e100831.

11. Brück K, Stel VS, Gambaro G, et al. CKD prevalence varies across the European general population. J Am Soc Nephrol 2016; 27(7): 2135-2147.

12. James MT, Hemmelgarn BR, Wiebe N, et al. Glomerular filtration rate, proteinuria, and the incidence and consequences of acute kidney injury: a cohort study. Lancet 2010; 376(9758): 2096-2103.

13. Manns B, Hemmelgarn B, Tonelli M, et al. Population based screening for chronic kidney disease: cost effectiveness study. BMJ 2010; 341: c5869.

14. O'Callaghan CA, Shine B, Lasserson DS. Chronic kidney disease: a large-scale population-based study of the effects of introducing the CKD-EPI formula for eGFR reporting. BMJ Open 2011; 1(2): e000308.

15. de Lusignan S, Tomson C, Harris K, et al. Creatinine fluctuation has a greater effect than the formula to estimate glomerular filtration rate on the prevalence of chronic kidney disease. Nephron Clin Pract 2011; 117(3): c213-c224.

16. Hill NR, Lasserson D, Fatoba S, et al. The Oxford Renal (OxRen) cross-sectional study of chronic kidney disease in the UK. BMJ Open 2013; 3(12): e004265.

17. Myers GL, Miller WG, Coresh J, et al. Recommendations for improving serum creatinine measurement: a report from the Laboratory Working Group of the National Kidney Disease Education Program. Clin Chem 2006; 52(1): 5-18.

18. Levey AS, Bosch JP, Lewis JB, et al. A more accurate method to estimate glomerular filtration rate from serum creatinine: a new prediction equation. Modification of Diet in Renal Disease Study Group. Ann Intern Med 1999. 130(6): 461-470

19. Altman D, Machin D, Bryant T, Gardner M, eds. Statistics with confidence: confidence intervals and statistical guidelines. 2nd edn. London: BMJ Books, 2013

20. Wilson EB. Probable inference, the law of succession, and statistical inference. Journal of the American Statistical Association 1927; 22(158): 209-212.
21. Eriksen BO, Ingebretsen $\mathrm{OC}$. In chronic kidney disease staging the use of the chronicity criterion affects prognosis and the rate of progression. Kidney Int 2007; 72(10): 1242-1248

22. Hirst JA, Montes MDV, Taylor CJ, et al. Impact of a single eGFR and eGFRestimating equation on chronic kidney disease reclassification: a cohort study in primary care. Br J Gen Pract 2018; DOI: https://doi.org/10.3399/bjgp18X697937.

23. McFadden EC, Hirst JA, Verbakel JY, et al. Systematic review and metaanalysis comparing the bias and accuracy of the Modification of Diet in Renal Disease and Chronic Kidney Disease Epidemiology collaboration equations in community-based populations. Clin Chem 2018; 64(3): 475-485.

24. Fraser SDS, Aitken G, Taal MW, et al. Exploration of chronic kidney disease prevalence estimates using new measures of kidney function in the Health Survey for England. PLoS One 2015; 10(2): e0118676.

25. Office for National Statistics. Ethnic group by sex by age. 2011. https://www. nomisweb.co.uk/census/2011/dc2101ew laccessed 14 Jan 2020).

26. Oxfordshire Data Observatory. Ethnicity in Oxfordshire. 2010. http://www. southoxon.gov.uk/sites/default/files/DataProjSELG201005RacePaper_final.pdf laccessed 14 Jan 2020).

27. Hull S, Dreyer G, Badrick E, et al. The relationship of ethnicity to the prevalence and management of hypertension and associated chronic kidney disease. BMC Nephrol 2011; 12: 41

28. Mathur R, Dreyer G, Yaqoob MM, Hull SA. Ethnic differences in the progression of chronic kidney disease and risk of death in a UK diabetic population: an observational cohort study. BMJ Open 2018; 8(3): e020145.

29. Jadawji C, Crasto W, Gillies C, et al. Prevalence and progression of diabetic nephropathy in South Asian, white European and African Caribbean people with type 2 diabetes: a systematic review and meta-analysis. Diabetes Obes Metab 2019; 21(3): 658-673

30. Hill NR, Fatoba ST, Oke JL, et al. Global prevalence of chronic kidney disease a systematic review and meta-analysis. PLoS One 2016; 11(7): e0158765.

31. Office for National Statistics. LC5102EW - Highest level of qualification by age. 2011. https://uww.nomisweb.co.uk/census/2011/LC5102EW/ view/2092957703? rows=c_hlqpuk11\&cols=c_age (accessed 14 Jan 2020).

32. Zeng X, Liu J, Tao S, et al. Associations between socioeconomic status and chronic kidney disease: a meta-analysis. J Epidemiol Community Health 2018; 72(4): 270-279.

33. Aitken GR, Roderick PJ, Fraser S, et al. Change in prevalence of chronic kidney disease in England over time: comparison of nationally representative crosssectional surveys from 2003 to 2010. BMJ Open 2014; 4(9): e005480.

34. Murphy D, McCulloch CE, Lin F, et al. Trends in prevalence of chronic kidney disease in the United States. Ann Intern Med 2016; 165(7): 473-481.

35. Patel N, Golzy M, Nainani N, et al. Prevalence of various comorbidities among veterans with chronic kidney disease and its comparison with other datasets. Ren Fail 2016; 38(2): 204-208.

36. Delanaye P, Glassock RJ, De Broe ME. Epidemiology of chronic kidney disease: think (at least) twice! Clin Kidney J 2017; 10(3): 370-374.

37. Shardlow A, McIntyre NJ, Fluck RJ, et al. Chronic kidney disease in primary care: outcomes after five years in a prospective cohort study. PLoS Med 2016; 13(9): e1002128.

38. Kearns B, Gallagher H, de Lusignan S. Predicting the prevalence of chronic kidney disease in the English population: a cross-sectional study. BMC Nephrol 2013; 14: 49.

39. Coghill N, Garside L, Montgomery AA, et al. NHS health checks: a crosssectional observational study on equity of uptake and outcomes. BMC Health Serv Res 2018; 18(1): 238.

40. Dryden R, Williams B, McCowan C, Themessl-Huber M. What do we know about who does and does not attend general health checks? Findings from a narrative scoping review. BMC Public Health 2012; 12: 723. 